\title{
Scalar arguments of the mathematical functions defining molecular and turbulent transport of heat and mass in compressible fluids
}

\author{
By ANDREW S. KOWALSKI I,2,* and DANIEL ARGÜESO ${ }^{1}, \quad{ }^{1}$ Departamento de Física Aplicada, \\ Facultad de Ciencias, Universidad de Granada, Avenida Fuentenueva, S/N, 18071 Granada, Spain; ${ }^{2}$ Centro Andaluz \\ de Medio Ambiente (CEAMA), 18006 Granada, Spain
}

(Manuscript received 4 December 2010; in final form 21 July 2011)

\begin{abstract}
The advection-diffusion equations defining control volume conservation laws in micrometeorological research are analysed to resolve discrepancies in their appropriate scalar variables for heat and mass transport. A scalar variable that is conserved during vertical motions enables the interpretation of turbulent mixing as 'diffusion'. Gas-phase heat advection is shown to depend on gradients in the potential temperature $(\theta)$, not the temperature $(T)$. Since conduction and radiation depend on $T$, advection-diffusion of heat depends on gradients of both $\theta$ and $T$. Conservation of $\theta$ (the first Law of Thermodynamics) requires including a pressure covariance term in the definition of the turbulent heat flux. Mass advection and diffusion are universally agreed to depend directly on gradients in the gas 'concentration' $(c)$, a nonetheless ambiguous term. Depending upon author, $c$ may be defined either as a dimensionless proportion or as a dimensional density, with non-trivial differences for the gas phase. Analyses of atmospheric law, scalar conservation and similarity theory demonstrate that mass advection-diffusion in gases depends on gradients, not in density but rather in a conserved proportion. Flux-tower researchers are encouraged to respect the meteorological tradition of writing conservation equations in terms of scalar variables that are conserved through simple air motions.
\end{abstract}

\section{Introduction}

Estimations of land-atmosphere exchange via micrometeorological 'flux tower' measurements - applying boundary-layer conservation concepts to interpret surface forcing-have become widespread in recent years. Much of such research is motivated by international commitments to identify, quantify, protect and enhance sinks of greenhouse gases (UN Framework Convention on Climate Change, 1997). Tower sites now number in the hundreds (Baldocchi, 2008), and are dedicated principally to measuring turbulent exchange processes. Since exchanges are not always characterized by turbulent transport, surface interactions are assessed as the lower boundary forcing a local volume about the tower, using a boundary-layer budget equation (Sorensen Geernaert et al., 1998; Finnigan, 2008).

\footnotetext{
* Corresponding author. e-mail: andyk@ugr.es DOI: $10.1111 /$ j.1600-0889.2011.00579.x
}

The advection-diffusion equation specifies spatio-temporal fluctuations in some scalar $\zeta$ within a boundary-layer control volume, generally written as

$$
\begin{aligned}
& \frac{\partial \zeta}{\partial t}+u_{i} \frac{\partial \zeta}{\partial x_{i}}=\frac{\partial}{\partial x_{i}}\left(K_{\zeta} \frac{\partial \zeta}{\partial x_{i}}\right)+J_{\zeta} \\
& \text { I II }
\end{aligned}
$$

Here, $u_{i}$ is the velocity, $K_{\zeta}$ the molecular transport coefficient and $J_{\zeta}$ a source term (Stull, 1988, eq. 3.2.4-3.2.6). The material derivative $\frac{D \zeta}{D t}$, as the sum of local change (I) and advection (II), is forced by the convergence in molecular fluxes (III) and any 'body' source term that may act (IV; 'body' effects act at the centre of mass, requiring no surface contact). Although consensus exists regarding the form of eq. (1), discrepancies abound regarding the appropriate form of the scalar-whose spatial and temporal fluctuations determine the relevant storage and transport processes involved-to substitute for $\zeta$.

Scientists inconsistently define the scalar arguments for mathematical functions expressing gas-phase transport of both heat and mass. For trace gas transport, the scalar $\zeta$ in eq. (1) might be substituted by constituent density $\left(\rho_{\mathrm{c}}\right.$; e.g. Businger, 1986; 
Stull, 1988) or by a dimensionless proportion (Baldocchi et al., 1988; Stull, 1988; Struthers et al., 2007; Font et al., 2010) such as the specific humidity $(q)$ or mixing ratio $(c)$. Even Fick's law might define molecular diffusion as proportional to dimensional (Sutton, 1953; Arya, 1988; Oke, 1990; Wyngaard, 1990) or dimensionless (Batchelor, 1967; Wyngaard, 1984; Holton, 1992; Bird et al., 2002) 'concentration' gradients. Similar discrepancies exist among micrometeorological texts regarding heat transport, with the scalar $\zeta$ specified as either the temperature ( $T$; Foken, 2008) or potential temperature $(\theta ;$ Stull, 1988). Since the left-hand side of eq. (1) defines material changes in the scalar $\zeta$, these variable definitions are clearly incompatible in the context of the resolved source term, highlighting the need to identify the appropriate scalar argument for each mass- and heat-transfer mechanism and reject unsuitable candidates.

This work aims to establish conclusively the scalar arguments of the mathematical functions defining (turbulent and non-turbulent) advection and molecular diffusion for heat and scalar exchanges in the atmosphere. In the following section, we note that the interpretation of turbulent-scale advection as analogous to 'diffusion' requires that the scalar $\zeta$ be conserved during vertical motions (expansion/compression). For heat transport, subsequent thermodynamic analyses show that the conservation equation is best defined in terms of spatio-temporal fluctuations in $\theta$, albeit with molecular conduction defined by gradients in $T$. Finally for scalar transport, we find indications from hydrostatic analysis, similarity theory and the gas law all in favour of a dimensionless definition of the scalar $\zeta$. The paper concludes promoting the use of conserved variables, consistent with longstanding meteorological tradition as applied to the boundary layer (Rossby, 1932; Betts and Albrecht, 1986).

\section{Turbulent 'diffusion' in compressible fluids}

Since gas molecules have separation distances large enough to act as if unaffected by intermolecular forces, except during relatively infrequent 'collisions', the compressibility of air and other gases must be considered when addressing conservation principles. For some classes of atmospheric motions, conservation of momentum can be very closely approximated (and vastly simplified) by assuming an incompressible version of the continuity equation (Mahrt, 1986). However, the conservation context of such an incompressibility assumption is critical to its validity, and it is not justified when attempting to express conservation of energy or mass in the gas phase. Rather, it is convenient to write an expression of conservation for a variable that is unaffected by expansion and compression, and this is particularly so when the objective is to characterize turbulent 'diffusion'.

In the context of boundary-layer conservation equations, the equivalent expression of advection in 'flux form' (Stull, 1988)_defining turbulent-scale advection in terms of an eddy covariance-requires an incompressibility assumption. Using calculus's product rule, eq. (1) can be rewritten exactly as

$$
\begin{aligned}
& \frac{\partial \zeta}{\partial t}+\frac{\partial}{\partial x_{i}} u_{i} \zeta-\zeta \frac{\partial u_{i}}{\partial x_{i}}=\frac{\partial}{\partial x_{i}}\left(K_{\zeta} \frac{\partial \zeta}{\partial x_{i}}\right)+J_{\zeta} \\
& \begin{array}{lllll}
\text { I } & \text { IIa } & \text { IIb } & \text { III } & \text { IV }
\end{array}
\end{aligned}
$$

Of the two terms now defining advection, term IIa is the divergence of a kinematic flux $\left(u_{i} \zeta\right)$-such as that quantifiable on small scales in terms of an eddy covariance-and term IIb quantifies the effects of compressibility. The estimation of term IIb might be problematic in general, but it can be neglected if the scalar $\zeta$ is conserved during expansion and compression. Then, similar interpretations can be used for the advective (IIa) and molecular (III) down-gradient fluxes whose convergence/divergence can enrich/deplete $\zeta$ locally. Such a conserved variable is effectively 'diffused' by advective processes such as (small-scale) turbulent mixing, which may therefore be written equivalently as either term II of eq. (1) or term IIa of eq. (2). The following sections present analyses that demonstrate advantages of using conserved scalars when defining advective and diffusive transport of heat and mass in compressible fluids.

\section{Scalar arguments of functions defining atmospheric heat transport}

\subsection{Convective heat exchange in the atmosphere}

Broad-sense convection includes atmospheric motions such as buoyancy-driven flows (strict-sense convection) and advection; the following analyses apply to all classes of convection, including turbulent 'diffusion'. Convection differs from conduction and radiation in terms of its relation to thermodynamic law, and this is especially important for compressible media like the atmospheric boundary layer.

The thermodynamic peculiarity of convection is highlighted first by its significance only in an Eulerian framework, where variable fluid properties are examined in a fixed volume of interest as is relevant to tower measurements. Thermodynamic laws govern systems constituted, not by Eulerian volumes with varying matter properties, but rather by identifiable pieces of matter corresponding to a Lagrangian fluid specification (Batchelor, 1967). Convection cannot bring about heat exchange in a purely Lagrangian framework, and in fact does not constitute thermal contact between thermodynamic systems. Recognizing this is critical for specifying convective heat exchange between thermodynamic systems at differing pressures, as demonstrated by the following example.

Let us consider two successive, isothermal atmospheric layers with equal temperature $(T)$, constituting two thermodynamic systems. Initially in thermal equilibrium, these two systems would exchange no heat via molecular or radiative processes, according to the zeroth Law of Thermodynamics. However, if subjected to the advective process of turbulent mixing they 
would tend away from thermal equilibrium and towards an adiabatic lapse rate. Such an adjustment of the thermal profile is possible only when drawing upon (turbulent kinetic) energy reserves to overcome static stability. The internal energy of air parcels transported vertically changes as they perform or receive work, prior to realizing thermal contact and exchanging heat (via local, molecular conduction). For compressible fluids the shortcoming of eq. (1) as an expression of the first Law of Thermodynamics-when substituting $T$ for $\zeta$-is precisely the lack of a work term modulating internal energy. However, rather than adding a source/sink (vertical-velocity-dependent term IV), tradition in meteorology defines a new state variable that is conserved through such processes and in the absence of thermal contact.

The above thermodynamic example demonstrates clearly the potential for hazard when attempting to include (Eulerian) advection in the application of (Lagrangian) conservation laws. Although the zeroth Law of Thermodynamics identifies $T$ as the scalar whose gradient determines spontaneous heat transport for thermodynamic systems in thermal contact, convective heat transfer does not fit such a framework and is in fact determined by a different scalar. Specifically, the potential temperature $(\theta)$ is the scalar that determines convective heat transfer in the dry boundary layer, and whose material derivative is relevant to the first Law of Thermodynamics for advective processes (Rossby, 1932). This material derivative includes not only the spatial derivatives defining advection, but also the time rate of local change ( $\theta$ is immune, e.g. to the compression caused by piling up on the air column aloft). However, this does not affect the fundamental dependencies of molecular and radiative heat exchanges on $T$ differences, as specified by the zeroth Law of Thermodynamics. This distinction between the scalar arguments for functions defining heat exchange processes has been recognized previously (Ha and Mahrt, 2003), but not explicitly put forth in terms of boundary-layer budget equations, to the authors' knowledge.

\subsection{The boundary-layer thermodynamic equation}

The proper form of the thermodynamic equation for the atmosphere requires the use of two thermodynamic variables, both the material derivative of $\theta$ determining local storage and convective heat exchange, and also gradients in $T$ governing molecular conduction. To demonstrate this, we begin with the first Law of Thermodynamics expressed as dividing diabatic effects (applying specific heat, $q$ ) into those detectable as $T$ changes at constant pressure ( $p$ ) and those related to pressure change (Petty, 2008, eq. 5.85). In combination with the gas law, this takes the form

$\mathrm{d} q=C_{p} \mathrm{~d} T-R T \frac{\mathrm{d} p}{p}$.

When (3) is divided by $\left(C_{p} T\right)$ it can be compared with the differential form of the definition of the potential temperature $(\theta)$

$\frac{\mathrm{d} \theta}{\theta}=\frac{\mathrm{d} T}{T}-\frac{R}{C_{p}} \frac{\mathrm{d} p}{p}$,

to deduce that increments of heat added (per unit mass) lead directly to increments in $\theta$ :

$\mathrm{d} q=C_{p} T \frac{\mathrm{d} \theta}{\theta}$.

In an advection-diffusion context, this means that the material derivative (storage plus advection) of the potential temperature is related directly to the diabatic heating rate via

$\frac{\partial \theta}{\partial t}+u_{i} \frac{\partial \theta}{\partial x_{i}}=\left(\frac{10^{5}}{p}\right)^{\frac{R}{C_{p}}} \frac{1}{C_{p}}\left\{-\frac{\partial}{\partial x_{i}}\left(-K_{T} \frac{\partial T}{\partial x_{i}}+J_{T}\right)\right\}$,

where $p$ is the pressure in S.I. units (Pa). In (6), the storage (I) and advection (II) terms are forced by the convergences of molecular conduction and the radiative flux $\left(J_{T}\right)$, both of which are determined by temperature; latent heating is ignored here.

The importance of using the proper form of (6), versus traditionally published versions, depends upon both its intended use/interpretation and also atmospheric conditions. This hybrid thermodynamic equation combines approaches based wholly either on $T$ (Foken, 2008) or on $\theta$ (Stull, 1988). The former neglects the effects of adiabatic pressure adjustments on advective transport, as may be appropriate near the surface where the magnitude of $T$ gradients (degrees per metre) and fluctuations are often large enough that the effects of adiabatic compression might be practically neglected. The latter appears to violate the zeroth Law of Thermodynamics governing molecular exchanges; however, since the difference between $\frac{\partial T}{\partial z}$ and $\frac{\partial \theta}{\partial z}$ is practically non-divergent $\left(g / C_{\mathrm{p}}\right)$, substituting $\theta$ for $T$ in the molecular term of (6) can be justified when assessing local heating. Nonetheless, the correct version of (6) may be critical for modelling nocturnal boundary layers, since for weak to moderate stability - with lapse rates between isothermal and adiabatic-vertical turbulent transport of heat opposes that by molecular and radiative processes (Fig. 1).

However useful it may be for modelling, eq. (6) is not the most directly applicable expression for interpreting measurements. Although the $\theta$ covariance has previously been recognized as defining the turbulent heat flux, despite what is implied in some publications (Kim and Mahrt, 1992; Moraes et al., 2004) no instruments exist that are capable of directly measuring turbulent fluctuations in $\theta$. Thus, a different thermodynamic construct can be appropriate for adapting eq. (6) to experimental data, and is put forth in the following derivation.

\subsection{The turbulent heat flux}

When (3) is scaled by air density $(\rho)$ and such diabatic forcings are correlated with vertical velocity fluctuations, it is seen that the turbulent heat flux $(H)$ must include a pressure-covariance 


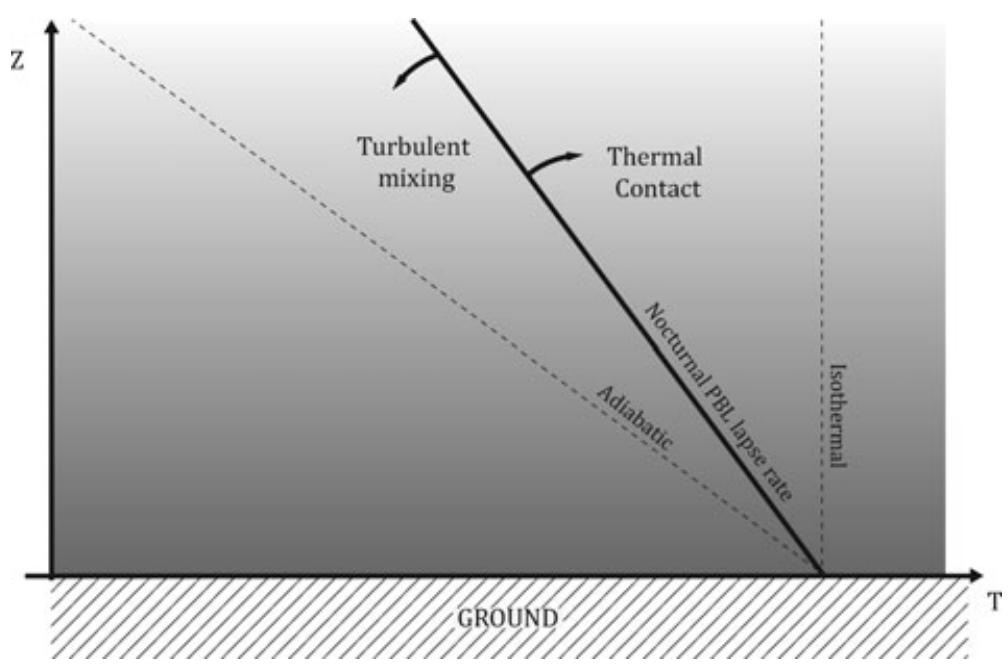

Fig. 1. The effects of different heat exchange processes on the lapse rate of a weakly stable planetary boundary layer (PBL). Advective heat exchange (turbulent mixing) is determined by gradients in $\theta$ and so pushes the lapse rate towards an adiabatic profile, whereas heat exchanges by molecular conduction and radiation (representing thermal contact) are driven by gradients in $T$ and cause the lapse rate to vanish.

turbulent transport term, as

$H=\rho C_{p} \overline{w^{\prime} T^{\prime}}-\overline{w^{\prime} p^{\prime}}$.

This definition is consistent with conclusions drawn from the second Law of Thermodynamics:

(1) that adiabatic processes are isentropic;

(2) that entropy is the thermodynamic scalar intensity whose fluctuations are proportional to diabatic forcing, and therefore whose covariance with vertical velocity should be used to represent the vertical flux of sensible heat; and

(3) that entropy fluctuations are proportional to the $T$ and $p$ fluctuations represented in eq. (7).

Traditional micrometeorological expressions for the sensible heat flux as $H=\rho C_{p} \overline{w^{\prime} T^{\prime}}$ account only for $T$ changes induced by heat exchange, neglecting the thermodynamic role of (dynamic) pressure fluctuations.

The final term in eq. (7), $\overline{w^{\prime} p^{\prime}}$ has received attention in the context of the turbulent kinetic energy budget (Wyngaard and Coté, 1971; Wyngaard et al., 1994), but has been largely neglected as contributing to the heat flux. Dimensional analysis confirms that the product of velocity (length/time) and pressure (force/area) represents an energy flux density, with units $\left(\mathrm{m} \mathrm{s}^{-1} \mathrm{~Pa}\right.$ ) exactly equivalent to $\mathrm{W} \mathrm{m}^{-2}$. Experimental determinations of $\overline{w^{\prime} p^{\prime}}$ are scarce-initial measurements suggest an order of magnitude of $5 \mathrm{~W} \mathrm{~m}^{-2}$ (Zhang et al., 2011) — but it may constitute a non-negligible fraction of the surface energy budget for certain situations (Sun et al., 1995; Massman and Lee, 2002).

To summarize this section regarding energy conservation principles, the scalar arguments of boundary-layer heat exchange functions vary according to the process under consideration. While molecular and radiative heat exchanges depend on differences in the temperature in accordance with the zeroth Law of Thermodynamics, the potential temperature $(\theta)$ is the relevant scalar determining heat transport by convection (including turbulent-scale advection) in compressible fluids. Turbulent fluctuations in $\theta$ can be decomposed into those associated with changes in temperature and/or varying (dynamic) pressure. The overall magnitude and importance of the latter remain open to investigation.

\section{Scalar arguments of atmospheric mass transport functions}

The advection-diffusion equation for a trace constituent is often specified as a first step in defining transport processes for the scalar concentration $(c)$ :

$$
\frac{\partial c}{\partial t}+u_{i} \frac{\partial c}{\partial x_{i}}=\frac{\partial}{\partial x_{i}}\left(K_{c} \frac{\partial c}{\partial x_{i}}\right)+J_{c}
$$

I II III IV

where $K_{C}$ is the molecular diffusivity defined by Fick's law, and $J_{C}$ represents a scalar source/sink term. As in the thermodynamic analogy, terms II and III specify advective/diffusive transport mechanisms directly associated with $c$ gradients in the fluid. The material derivative $\frac{D c}{D t}$, defined by the sum of local change (I) and advection (II), is subject to forcing by the convergence in molecular diffusion (III) and any 'body' source term (IV). Flux-tower research aims to assess $\frac{D c}{D t}$ aerodynamically within a control volume, and so infer gas exchange at the surface where forcing terms are not assumed to be negligible (Finnigan, 2008). Given Fourier's requirement for dimensional homogeneity among terms in physical equations (Buckingham, 1914), the dimensions specified for the scalar $c$ in eq. (8) influence the nature of the material derivative and relevant surface forcing processes.

Despite the agreed form for eq. (8), inconsistency remains regarding its dimensions and hence fundamental meaning for the (compressible) gas phase. This equation derives from a thermodynamic analogy, substituting $c$ for $T$, Fick's law for Fourier's and scalar sources for the effects of heating. Yet the analogy is incomplete; whereas the zeroth Law of Thermodynamics 
establishes $T$ as the thermodynamic variable whose differences determine heat exchange, the scalar $c$ whose gradients define these processes is not uniquely defined. Rather, atmospheric literature admits conflicting definitions for $c$, grouped generally into two paradigms as either

(1) 'absolute' $\left(c_{a}\right)$ - in air chemistry (Seinfeld and Pandis, 1998) and sometimes micrometeorology (Businger, 1986; Finnigan et al., 2003), gas-phase concentration is a synonym for constituent density $\left(\rho_{\mathrm{c}}\right)$, quantifying the constituent mass (or moles, with a trivial difference of scaling by molecular mass) per unit of fluid volume; or

(2) 'relative' $\left(c_{r}\right)$ —climatologists such as those forming the IPCC (Forster et al., 2007) and sometimes micrometeorologists (Kaimal and Finnigan, 1994) define gas-phase concentration with dimensionless units as the amount of constituent gas per amount of fluid considered.

The dimensionality of $c$ is sometimes left undefined (Lee, 1998; Yi et al., 2000) or inconsistent within the same publication (Mammarella et al., 2007; van Gorsel et al., 2007).

For liquids the direct correspondence between volume and total fluid amount precludes any inconsistency. Therefore, resolving ambiguity and establishing general fluid law must proceed from analysis of the gas phase, where differences between these two paradigms can be of fundamental importance. The following analyses aim to identify the appropriate $c$ paradigm for gas-phase diffusion, advection and scalar conservation. Considering the flux-tower objective of estimating surface forcing by resolving eq. (8), its terms are examined in the remainder of this section in an order chosen based on consequence and for the purpose of concision.

\subsection{Molecular diffusion defined by gradients in 'concentration'}

A survey of the literature offers contradictions concerning the meaning of 'concentration' in Fick's law (in term III of eq. 8). Both textbooks (Sutton, 1953; Arya, 1988; Oke, 1990; Campbell and Norman, 1998; Nobel, 2005) and research papers addressing diffusion (Bres and Hatzfeld, 1977; Businger, 1986; Wyngaard, 1990; Pihlatie et al., 2007; Pingintha et al., 2010) have expressed Fick's law for the gas phase explicitly in terms of $c_{a}$ (i.e. constituent density, or $\rho_{\mathrm{c}}$ ). Conversely, texts on fluid dynamics and transport phenomena (Batchelor, 1967; Holton, 1992; Bird et al., 2002) and other researchers (Wyngaard, 1984; Wyngaard and Brost, 1984; Baldocchi et al., 1988; Davidson and Trumbore, 1995; Fierer et al., 2005; Kim et al., 2007) specify Fickan diffusion in terms of gradients in $c_{r}$. Since the correct choice of $c$ for defining Fick's law matters fundamentally only in the compressible case, perhaps it is no surprise that this conflict can be resolved by examining diffusion in light of the vertical structure of the atmosphere and one of its most basic physical laws.
Prior to describing the atmosphere in motion, it is essential to understand its hydrostatics, and so let us examine the static case and specifically the implications arising from these competing definitions for Fick's law. For the 1976 standard troposphere, the relative concentrations $\left(c_{r}\right)$ of all gases are constant (e.g. 314 ppm $\mathrm{CO}_{2}$ ) whereas the absolute air concentration $\left(c_{a}\right.$; or $\left.\rho_{\mathrm{c}}\right)$ decreases continually with height (NOAA et al., 1976). In the relative paradigm, all $c_{r}$ gradients are zero for this well-mixed case and there is no net gas transport by molecular diffusion. According to the absolute paradigm however, the negative vertical gradient in $c_{\mathrm{a}}$ (or $\rho_{\mathrm{c}}$ ) would seem to imply upward diffusion of every gas. Such upward gas transport by molecular processes is impossible because it would break the balance between gravity and the vertical pressure gradient for this static atmosphere. Thus, the meaning of concentration $(c)$ in term III of eq. (8) must be relative and dimensionless, and this conclusion from hydrostatic analysis will presently be confirmed by an analysis of isobaric heat conduction.

Let us now consider the case of molecular transport for the case of steady-state heat transfer down a temperature gradient, as in a thin air layer between two parallel, inert plates of differing temperatures (Fig. 2). If the air pressure on each plate is the same,

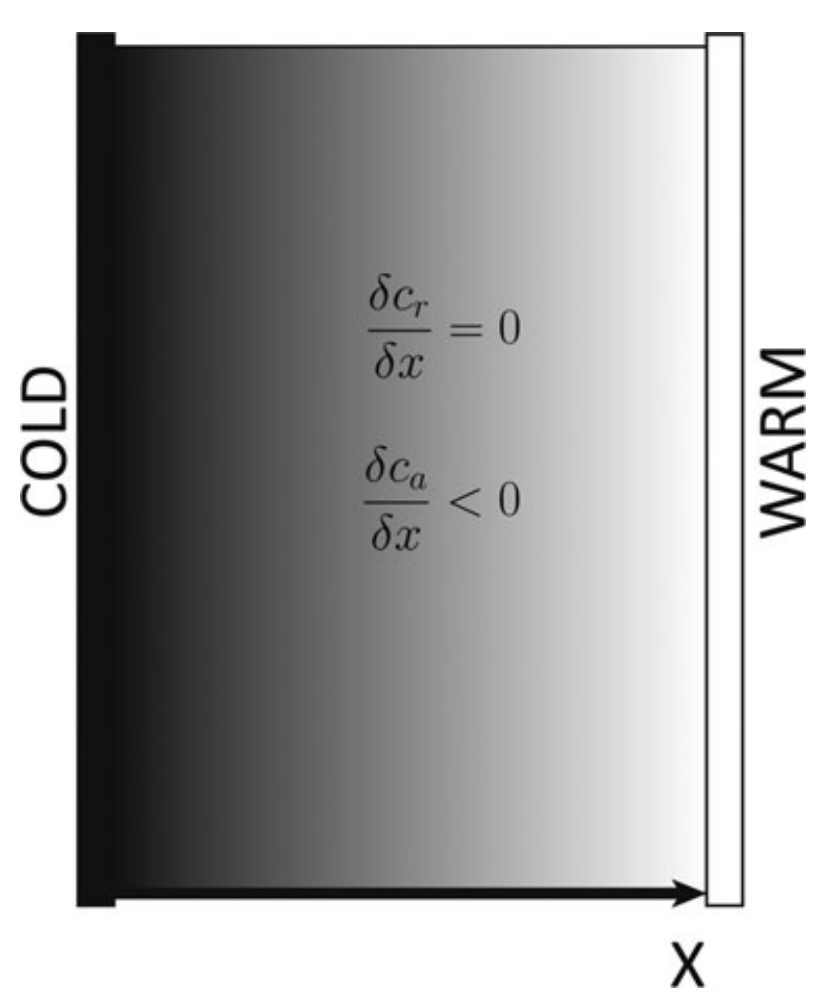

Fig. 2. Steady-state heat exchange across a thin layer of air by molecular conduction from a warm plate to a cold plate at equal pressures. The relative concentration (proportion) of all gas constituents is everywhere constant, whereas gradients in the absolute concentration (density) exist for every constituent in accordance with the ideal gas law. 
then the ideal gas law informs us that the air in contact with the cold plate will be denser. This is true not only for the gas mixture defined as air, but also for every one of its constituents, such as $\mathrm{CO}_{2}$. That is, a gradient in the absolute concentration $c_{a}$ (or $\rho_{c}$ ) exists, and if this were the variable appropriate for substitution into eq. (8), it would seem to imply diffusion towards the warmer plate, which does not occur. This case of thermal conduction is further evidence that the relative paradigm must be the correct one for compressible fluids - and therefore in general—when describing molecular diffusion.

\subsection{The material derivative and body forcing terms}

Material changes-represented by terms I and II of eq. (8) for mixture and constituent state variables are governed by the gas law, with profoundly different implications for $c_{a}$ (or $\rho_{\mathrm{c}}$ ) versus $c_{r}$. If the scalar in eq. (8) is $c_{r}$, then the material derivative $\frac{D c}{D t}$ is zero (e.g. conservation of the mixing ratio is demonstrated in the Appendix). If the scalar in eq. (8) is $c_{a}$ (or $\rho_{\mathrm{c}}$ ), then the material derivative $\frac{D c}{D t}$ is subject to surface forcing dominated by exchanges of heat and water vapour (Webb et al., 1980). In this case, detecting trace gas exchange requires adjusting the aerodynamically assessed material derivative in eq. (8) to account for 'WPL effects' on the control volume mass balance (Finnigan, 2009), confounding the physical interpretation of the relationship between the material derivative and gas exchange. Although not explicitly so stated, the 'eddy flux density' in eq. (1) of the (Finnigan, 2009) mass balance must not be WPL corrected (and covariances involving $c_{r}$ from a closed-path IRGA must in fact be reverse WPL corrected to calculate such density covariances). Moreover, experimental estimates of $\frac{\partial c_{a}}{\partial t}$ (for term I) and $\frac{\partial c_{a}}{\partial x}$ (for term II) using a lone gas analyser with multiple sample inlets require additional temperature measurements for every inlet. By contrast, if the scalar in eq. (8) is $c_{r}$, then exchange of the relevant trace gas can be determined directly from the material derivative (terms I and II), assessed using traditional methods and applying WPL corrections when turbulent fluxes are determined using open-path IRGAs.

As discussed in Section 2, for scalar transport the very interpretation of an eddy covariance as (turbulent-scale) advectionand thus the lone complement to storage in forming the material derivative-requires the use of the continuity equation in its incompressible form. If the scalar represented in eq. (8) were $c_{a}$ (or $\rho_{\mathrm{c}}$ ), then with varying temperature such an incompressibility assumption would lead to a false mass conservation expression and unacceptable errors (for $\mathrm{CO}_{2}$ exchange, of order $\left.1 \mu \mathrm{mol} \mathrm{m}{ }^{-2} \mathrm{~s}^{-1}\right)$ in boundary-layer scalar constituent budgets (Kowalski and Serrano-Ortiz, 2007). Only when using $c_{r}-\mathrm{a}$ conserved variable immune to compressibility effects—can we proceed to derive from the eddy covariance a turbulent-scale advection term analogous to diffusion, without restriction to the (trivial) isothermal case.
The choice of $c_{r}$ to describe boundary-layer processes is further supported by other fundamental micrometeorological principles underlying flux-gradient (K-theory) models of advective transport. There is no justification for empirically relating mass transport to gradients in $c_{\mathrm{a}}$ (or $\rho_{\mathrm{c}}$ ), which can be due largely to atmospheric pressure and temperature gradients not strictly related to the (turbulent or molecular) diffusion of matter. On the contrary, dimensional consistency is a prerequisite for the development of models based on physical similarity (Barenblatt, 2003), such as the flux-gradient description of turbulent mixing - defined long ago in terms of the relative concentration (Businger and Yaglom, 1971; Obukhov, 1971) —as analogous to Fick's law for the molecular case.

In addition to superficial/contact forcing by molecular transfer (Section 4.1), any 'body' forcing (term IV) can lead to concentration changes in eq. (8). For example, reacting monatomic and diatomic oxygen molecules represent a body source $\left(J_{C}>0\right)$ for ozone in the interior of the fluid. Generally, body sources are neglected for 'passive scalars' such as $\mathrm{CO}_{2}$ (Katul et al., 1996) on the basis that their sources/sinks exist only at the surface (Finnigan et al., 2003), supposing that $\frac{D c}{D t}=0$ in the interior of the fluid. However, as noted above $\frac{D c_{a}}{D t}$ is non-zero for diabatic processes including not only molecular conduction but also divergent radiative fluxes (greenhouse gas absorption) that can be important away from the surface. Simple vertical motions likewise lead to non-zero $\frac{D c_{a}}{D t}$ (see Appendix) due to associated expansion/compression. To avoid the existence of passive scalar body sources in the fluid interior, a relative definition of concentration is essential for the final term in eq. (8), just as for the other terms as outlined earlier.

\section{Conclusions}

For compressible media such as the atmosphere, the advection-diffusion equations applied to an Eulerian control volume should be expressed, not based on some absolute fluid property such as temperature or constituent density (Finnigan et al., 2003; Finnigan, 2004), but rather in terms of kinematic fluxes of a conserved variable.

Whereas temperature $(T)$ is the scalar argument for heat exchange functions due to thermal contact and thus obeying the zeroth Law of Thermodynamics, convective heat transfer in compressible fluids is determined by gradients in the potential temperature $(\theta)$. Conservation of $\theta$ is the most appropriate starting point for expressing the (dry) boundary-layer thermodynamic equation. As a consequence, the turbulent heat flux is defined in terms of the covariance of vertical winds, not only with $T$ but also with (dynamic) pressure.

In the gas phase, 'concentration' is a vague term whose variable definition can lead to errors in the interpretation of physical law. For the advection-diffusion equation, gas constituent conservation must be specified in terms of spatio-temporal fluctuations in the relative concentration (i.e. molar or mass fraction), 
specifying the time rate of change of a dimensionless quantity with units such as ppmv or $\mathrm{g} \mathrm{kg}^{-1}$, and not the constituent density. The classification of diffusion and advection as rates of change of dimensionless ratios-essential for compressible fluids (gases) yet inconsequential for incompressible liquids-can be considered valid for all fluids in general.

\section{Acknowledgments}

The authors received funding support from Andalusian regional government project GEOCARBO (P08-RNM-3721), the National Institute for Agrarian Research and Technology (INIA; SUM2006-00010-00-00), the Spanish flux-tower network CARBORED-ES (Science Ministry project CGL201022193-C04-02), and the European Commission collaborative project GHG Europe (FP7/2007-2013; grant agreement 244122). Credit for the Appendix goes to Dr. X. Lee of Yale University. Suggestions from Dr. P. Serrano-Ortiz and Dr. J. Sun helped to enhance the clarity and concision of this work.

\section{Appendix}

Conservation of the mixing ratio $(c)$, a relative concentration $\left(c_{r}\right)$ defined as the density ratio of a trace gas constituent $\left(\rho_{\mathrm{c}}\right)$ to dry air $\left(\rho_{\mathrm{d}}\right)$, can be shown in a few simple steps. The material derivative $\frac{D c}{D t}$ is defined according to the quotient rule of calculus as

$\frac{D c}{D t}=\frac{D\left(\rho_{c} / \rho_{d}\right)}{D t}=\frac{1}{\rho_{d}} \frac{D \rho_{c}}{D t}-\frac{\rho_{c}}{\rho_{d}^{2}} \frac{D \rho_{d}}{D t}$.

Supposing that all dry air constituents move at the same velocity (i.e. neglecting molecular diffusion), and substituting into eq. (A1) the (Lagrangian) continuity equation for both $\mathrm{CO}_{2}$

$\frac{D \rho_{c}}{D t}=-\rho_{c} \frac{\partial u_{i}}{\partial x_{i}}$

and dry air

$$
\frac{D \rho_{d}}{D t}=-\rho_{d} \frac{\partial u_{i}}{\partial x_{i}},
$$

the two terms on the right-hand side of eq. (A1) cancel out and the material derivative of the mixing ratio is shown to be zero (i.e. $c$ is conserved).

\section{References}

Arya, S. P. 1988. Introduction to Micrometeorology. Academic Press, San Diego.

Baldocchi, D. 2008. Breathing of the terrestrial biosphere: lessons learned from a global network of carbon dioxide flux measurement systems. Australian J. Bot. 56, 1-26.

Baldocchi, D. D., Hicks, B. B. and Meyers, T. P. 1988. Measuring biosphere-atmosphere exchange of biologically-related gases with micrometeorological methods. Ecology 69, 1331-1340.
Barenblatt, G. I. 2003. Scaling. Cambridge University Press, Cambridge. Batchelor, G. K. 1967. An Introduction to Fluid Dynamics. Cambridge University Press, Cambridge.

Betts, A. K. and Albrecht, B. A. 1986. Conserved variable analysis of the convective boundary layer thermodynamic structure over the tropical oceans. J. Atmos. Sci. 44, 83-99.

Bird, R. B., Stewart, W. E. and Lightfoot, E. N. 2002. Transport Phenomena. John Wiley \& Sons, Cambridge.

Bres, M. and Hatzfeld, C. 1977. Three-gas diffusion: experimental and theoretical study. Eur. J. Physiol. 371, 227-233.

Buckingham, E. 1914. On physically similar systems: illustrations of the use of dimensional equations. Phys. Rev. 4, 345-376.

Businger, J. A. 1986. Evaluation of the accuracy with which dry deposition can be measured with current micrometeorological techniques. J. Clim. Appl. Meteorol. 25, 1100-1124.

Businger, J. A. and Yaglom, A. M. 1971. Introduction to Obukhov's paper on 'Turbulence in an atmosphere with a non-uniform temperature'. Bound.-Layer Meteorol. 2, 3-6.

Campbell, G. S. and Norman, J. M. 1998. Environmental Biophysics. Springer-Verlag, New York.

Davidson, E. A. and Trumbore, S. E. 1995. Gas diffusivity and production of $\mathrm{CO}_{2}$ in the deep soils of the eastern Amazon. Tellus 47B, 550-565.

Fierer, N., Chadwick, O. A. and Trumbore, S. E. 2005. Production of $\mathrm{CO}_{2}$ in soil profiles of a California annual grassland. Ecosystems $\mathbf{8}$, 412-429.

Finnigan, J. J. 2004. A re-evaluation of long-term flux measurement techniques. Part II: Coordinate systems. Bound.-Layer Meteorol. 113, $1-41$.

Finnigan, J. 2008. An introduction to flux measurements in difficult conditions. Ecol. Appl. 18, 1340-1350.

Finnigan, J. J. 2009. Response to comment by Dr. A.S. Kowalski on "The storage term in eddy flux calculations". Agri. Forest Meteorol. 149, 725-729.

Finnigan, J. J., Clement, R., Malhi, Y., Leuning, R. and Cleugh, H. A. 2003. A re-evaluation of long-term flux measurement techniques. Part I: averaging and coordinate rotation. Bound.-Layer Meteorol. 107, $1-48$.

Foken, T. 2008. Micrometeorology. Springer-Verlag, Berlin Heidelberg. Font, A., Morguí, J.-A., Curcoll, R., Pouchet, I., Caals, I. and coauthors. 2010. Daily carbon surface fluxes in theWest Ebre (Ebro) watershed from aircraft profiling on late June 2007. Tellus 62B, 427-440.

Forster, P., Ramaswamy, V., Artaxo, P., Berntsen, N. L., Betts, R. and co-authors. 2007. Changes in atmospheric constituents and in radiative forcing. In: Climate Change 2007: The Physical Science Basis. Contribution of Working Group I to the Fourth Assessment Report of the Intergovernmental Panel on Climate Change eds. Solomon, S., D. Qin, M. Manning, Z. Chen, M. Marquis et al.). Cambridge University Press, Cambridge, United Kingdom and New York, NY, USA.

van Gorsel, E., Leuning, R., Cleugh, H. A., Keith, H. and Suni, T. 2007. Nocturnal carbon efflux: reconciliation of eddy covariance and chamber measurements using an alternative to the $\mathrm{u}^{*}$-threshold filtering technique. Tellus 59B, 397-403.

Ha, K.-J. and Mahrt, L. 2003. Radiative and turbulent fluxes in the nocturnal boundary layer. Tellus 55A, 317-327.

Holton, J. R. 1992. An Introduction to Dynamic Meteorology. Academic Press, San Diego. 
Kaimal, J. C. and Finnigan, J. J. 1994. Atmospheric Boundary Layer Flows Their Measurement and Structure. Oxford University Press, New York.

Katul, G. G., Finkelstein, P. L., Clarke, J. F. and Ellestad, T. G. 1996. An investigation of the conditional sampling method used to estimate fluxes of active, reactive, and passive scalars. J. Appl. Meteorol. 35, 1835-1845.

Kim, J. and Mahrt, L. 1992. Simple formulation of turbulent mixing in the stable free atmosphere and nocturnal boundary layer. Tellus $\mathbf{4 4 A}$, 381-394.

Kim, Y., Masahito, U., Nakagawa, F., Tsunogai, U., Harazono, Y. and coauthors. 2007. Assessment of winter fluxes of $\mathrm{CO}_{2}$ and $\mathrm{CH}_{4}$ in boreal forest soils of central Alaska estimated by the profile method and the chamber method: a diagnosis of methane emission and implications for the regional carbon budget Tellus 59B, 223-233.

Kowalski, A. S. and Serrano-Ortiz, P. 2007. On the relationship between the eddy covariance, the turbulent flux, and surface exchange for a trace gas such as $\mathrm{CO}_{2}$. Bound.-Layer Meteorol. 124, 129-141.

Lee, X. 1998. On micrometeorological observations of surface-air exchange over tall vegetation. Agri. Forest Meteorol. 91, 39-49.

Mahrt, L. 1986. On the shallow motion approximations. J. Atmos. Sci. 43, 1036-1044.

Mammarella, I., Kolari, P., Rinne, J., Keronen, P. and Pumpanen, J. 2007. Determining the contribution of vertical advection to the net ecosystem exchange at Hyytiälä forest, Finland. Tellus 59B, 900-909.

Massman, W. J. and Lee, X. 2002. Eddy covariance flux corrections and uncertainties in long term studies of carbon and energy exchanges. Agri. Forest Meteorol. 113, 121-144.

Moraes, O. L. L., Acevedo, O. C., da Silva, R., Magnago, R. and Siqueira, A. C. 2004. Nocturnal surface-layer characteristics at the bottom of a valley. Bound.-Layer Meteorol. 112, 159-177.

NOAA, NASA and USAF. 1976. U. S. Standard Atmosphere, 1976. U.S. Government Printing Office, Washington, DC.

Nobel, P. S. 2005. Physicochemical and Environmental Plant Physiology. Elsevier Academic Press, Amsterdam.

Obukhov, A. M. 1971. Turbulence in an atmosphere with a non-uniform temperature. Bound.-Layer Meteorol. 2, 7-29.

Oke, T. R. 1990. Boundary Layer Climates. University Press, Cambridge.

Petty, G. W., 2008. A First Course in Atmospheric Thermodynamics, Sundog, Madison.

Pihlatie, M., Pumpanen, J., Rinne, J., Ilvesniemi, H., Simojoki, A. and co-authors. 2007. Gas concentration driven fluxes of nitrous oxide and carbon dioxide in boreal forest soil. Tellus 59B, 458-469.

Pingintha, N., Leclerc, M. Y., Beasley, J. P., Zhang, G. J. and Senthong, C. 2010. Assessment of the soil $\mathrm{CO}_{2}$ gradient method for soil $\mathrm{CO}_{2}$ efflux measurements: comparison of six models in the calculation of the relative gas diffusion coefficient. Tellus 62B, 47-58.

Rossby, C.-G. 1932. Thermodynamics applied to air mass analysis. Meteorol. Papers 1, 1-41.

Seinfeld, J. H. and Pandis, S. N. 1998. Atmospheric Chemistry and Physics: From Air Pollution to Climate Change, John Wiley $\&$ Sons, New York.

Sorensen Geernaert, L. L., Geernaert, G. L., Granby, K. and Asman, W. A. H. 1998. Fluxes of soluble gases in the marine atmospheric surface layer. Tellus 50B, 111-127.

Struthers, H., Allan, W., Lowe, D. C. and Bhaskaran, B. 2007. A comparison of the transport of long-lived atmospheric trace gas species from two advection schemes incorporated into an atmospheric general circulation model. Tellus 59B, 685-697.

Stull, R. B. 1988. An Introduction to Boundary Layer Meteorology. Kluwer, the Netherlands.

Sun, J., Esbensen, S. K. and Mahrt, L. 1995. Estimation of surface heat flux. J. Atmos. Sci. 52, 3162-3171.

Sutton, O. G. 1953. Micrometeorology. McGraw-Hill Book Company, Inc., New York.

UN Framework Convention on Climate Change 1997. Kyoto Protocol to the United Nations Framework Convention on Climate Change, Kyoto, 23.

Webb, E. K., Pearman, G. I. and Leuning, R. 1980. Correction of flux measurements for density effects due to heat and water vapour transfer. Q. J. R. Meteorol. Soc. 106, 85-100.

Wyngaard, J. C. 1984. Towards convective boundary layer parameterization: a scalar transport module. J. Atmos. Sci. 41, 1959-1969.

Wyngaard, J. C. 1990. Scalar fluxes in the planetary boundary layer: theory, modeling, and measurement. Bound.-Layer Meteorol. 50, $49-75$.

Wyngaard, J. C. and Brost, R. A. 1984. Top-down and bottom-up diffusion of a scalar in the convective boundary layer. J. Atmos. Sci. 41, 102-112.

Wyngaard, J. C. and Coté, O. R. 1971. The budgets of turbulent kinetic energy and temperature variance in the atmospheric surface layer. $J$. Atmos. Sci. 28, 190-201.

Wyngaard, J. C., Siegel, A. and Wilczak, J. M. 1994. On the response of a turbulent-pressure probe and the measurement of pressure transport. Bound.-Layer Meteorol. 69, 379-396.

Yi, C., Davis, K. J., Bakwin, P. S., Berger, B. W. and Marr, L. C. 2000. Influence of advection on measurements of the net ecosystematmosphere exchange of $\mathrm{CO} 2$ from a very tall tower. J. Geophys. Res. 105, 9991-9999.

Zhang, J., Lee, X., Song, G., and Han, S., 2011, Pressure correction to the long-term measurement of carbon dioxide flux, Agri. Forest Meteorol. 151, 70-77. 